\title{
Complex chemistry with complex compounds
}

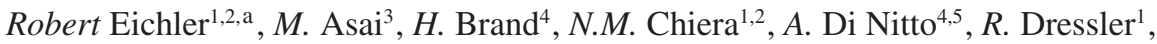 \\ Ch.E. Düllmann ${ }^{4,5,6}, J$. Even ${ }^{4,6}, F$. Fangli ${ }^{7}, M$. Goetz ${ }^{4,5,6}, H$. Haba ${ }^{8}$, W. Hartmann ${ }^{4}$, E. Jäger ${ }^{4}$, \\ D. Kaji ${ }^{8}, J$. Kanaya ${ }^{8}, Y$. Kaneya ${ }^{3}, J$. Khuyagbaatar ${ }^{4,6}, B . \mathrm{Kindler}^{4}, Y$ Komori $^{8}$, B. Kraus ${ }^{1,2}$, \\ J.V. Kratz ${ }^{5}, J . \mathrm{Krier}^{4}, Y . \mathrm{Kudou}^{8}, N . \mathrm{Kurz}^{4}, S$. Miyashita $^{3,9}, K$. Morimoto $^{8}, K$. Morita $^{8,10}$,

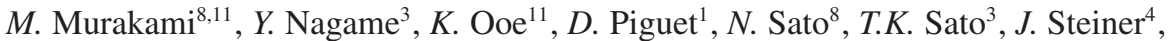 \\ $P$. Steinegger ${ }^{1,2}, T$. Sumita ${ }^{8}, M$. Takeyama ${ }^{8}, K$. Tanaka ${ }^{8}, T$. Tomitsuka ${ }^{11}, A$. Toyoshima ${ }^{3}$, \\ $K$. Tsukada ${ }^{3}, A$. Türler ${ }^{1,2}, I$. Usoltsev ${ }^{1,2}, Y$. Wakabayashi ${ }^{8}, Y$. Wang ${ }^{7}, N$. Wiehl $^{5,6}$, \\ $Y$. Wittwer ${ }^{1,2}, A$. Yakushev ${ }^{4,6}, S$. Yamaki ${ }^{8}, S$. Yano ${ }^{8}, S$. Yamaki ${ }^{8}$, and Z. Qin ${ }^{7}$ \\ ${ }^{1}$ Paul Scherrer Institute, 5232 Villigen, Switzerland \\ ${ }^{2}$ University of Bern, 3012 Bern, Switzerland \\ ${ }^{3}$ Advanced Science Research Center, Japan Atomic Energy Agency, Tokai, Ibaraki 319-1195, Japan \\ ${ }^{4}$ GSI Helmholtzzentrum für Schwerionenforschung GmbH, 64291 Darmstadt, Germany \\ ${ }^{5}$ Johannes Gutenberg-Universität Mainz, 55099 Mainz, Germany \\ ${ }^{6}$ Helmholtz-Institut Mainz, 55099 Mainz, Germany \\ ${ }^{7}$ Institute of Modern Physics Lanzhou, Chinese Academy of Sciences, 730000 Lanzhou, China \\ ${ }^{8}$ Nishina Center for Accelerator-Based Science, RIKEN, Wako, Saitama 351-0198, Japan \\ ${ }^{9}$ Hiroshima University, Kagamiyama, Higashi-Hiroshima 739-8526, Japan \\ ${ }^{10}$ Kyushu University, Higashi-Ku, Fukuoka, 812-8581, Japan \\ ${ }^{11}$ Niigata University, Niigata, Niigata 950-2181, Japan
}

\begin{abstract}
In recent years gas-phase chemical studies assisted by physical pre-separation allowed for the investigation of fragile single molecular species by gas-phase chromatography. The latest success with the heaviest group 6 transactinide seaborgium is highlighted. The formation of a very volatile hexacarbonyl compound $\operatorname{Sg}(\mathrm{CO})_{6}$ was observed similarly to its lighter homologues molybdenum and tungsten. The interactions of these gaseous carbonyl complex compounds with quartz surfaces were investigated by thermochromatography. Second-generation experiments are under way to investigate the intramolecular bond between the central metal atom of the complexes and the ligands addressing the influence of relativistic effects in the heaviest compounds. Our contribution comprises some aspects of the ongoing challenging experiments as well as an outlook towards other interesting compounds related to volatile complex compounds in the gas phase.
\end{abstract}

\section{Introduction}

About two thirds of the transactinide elements known today with $104 \leq Z \leq 111$ are expected to be typical members of the so-called transition metal series. These elements are known as typical metals and reveal an exceptionally low volatility in the elemental state - the lowest known amongst all elements of the periodic table. Hence, their gas-phase chemical investigation relies on the formation of volatile compounds. So far, only very stable

\footnotetext{
${ }^{a}$ Corresponding author: robert.eichler@psi.ch
}

(C) The Authors, published by EDP Sciences. This is an Open Access article distributed under the terms of the Creative Commons Attribution License 4.0 (http://creativecommons.org/licenses/by/4.0/). 
and simple inorganic compounds like oxides, oxohydroxides, halides or oxohalides of the transactinides Rf-Hs have been investigated proving their typical transition metal behaviour (see references $[1,2]$ for recent reviews). Pioneering experiments performed at the Berkeley Gas-filled Separator (BGS) at the Lawrence Berkeley National Laboratory (LBNL), USA between 2003 and 2005 investigated the possibility of in-situ formation of gas-phase complex compounds of group 4 elements of the periodic table $\mathrm{Zr}$ and $\mathrm{Hf}$ with the goal to investigate the corresponding compound formation also for element 104, rutherfordium (Rf) [3]. It was shown that pre-separated products of complete fusion reactions can be stopped in a carrier gas stream containing a reactive species suited as ligand for gas-phase complex formation. An in-situ formation of volatile species was observed serving as proof-of-principle and paving the way towards the investigation of new fragile compound classes of transactinides. Later in 2012-2013 the in-situ formation of volatile species of transition metal elements $\mathrm{Mo}, \mathrm{Tc}, \mathrm{Ru}, \mathrm{Rh}$ were observed produced as short-lived fission products recoiling out from targets of ${ }^{249} \mathrm{Cf}$ and ${ }^{238} \mathrm{U}$ at the TRIGA reactor of the Institute for Nuclear Chemistry at the University of Mainz, Germany, into a carrier gas stream containing carbon monoxide, CO $[4,5]$. Experiments at the TASCA separator at GSI Darmstadt, Germany [4-6] as well as at the Institute of Modern Physics, Lanzhou, China, confirmed these observations [7, 8] and demonstrated the formation of volatile species as well for recoiling nuclear fusion reaction products of transition metal elements of the sixth row of the periodic table: W, Re, Os, Ir. Chemical properties established for transition metals in CO containing chemical systems allowed identifying those volatile products likely as gas-phase carbonyl complexes. The metal-ligand bonding in carbonyl complexes is somewhat special, as the central atom stays in its zeroth oxidation state. The bonding emerges from electron pair donation from the $\mathrm{CO}$ molecules surrounding the central atom into its empty $d$ orbitals and subsequent $\pi$-back bonding due to overlapping of the thus filled $d$ orbitals with antibonding $\pi^{*}$-orbitals of CO, the latter loosening the $\mathrm{C}-\mathrm{O}$ bond, but strengthening the $\mathrm{M}-\mathrm{C}$ bond (see reference [9] for a review). The number of CO ligands is driven by the number of empty $d$ orbitals of the central atom. Thus the 18-electron rule applies, suggesting complexes with 18 electrons to be most stable configurations, e.g. $\mathrm{M}(\mathrm{CO})_{6}$ for group 6 elements and $\mathrm{M}(\mathrm{CO})_{5}$ for group 8 elements. Typically, the elements from odd group numbers form dimer or trimer complexes with $\mathrm{CO}$ bridges or additional intermetallic cluster bonds to stabilize themselves according to the 18-electron rule. This is obviously impossible for single atomic amounts as observed in these experiments. Thus the volatile species observed for the even-numbered group members could be attributed to predominant formation of the most stable configurations. However, the speciation of the volatile products of odd-numbered elements appears to be tentative.

\section{Group 6 carbonyl complex compounds}

Interestingly, already in 1999 predictions about the stability of hexacarbonyl complexes of group 6 elements and uranium were published [10] (see Table 1). Relativistic density functional theory was used to calculate the geometry and thermodynamic stability of these carbonyl species for Mo, W, and Sg indicating the hexacarbonyl species as the most stable ones obeying the 18 electron rule and suggesting the stability increase towards the heavier elements. This was attributed mainly to the increasing secondary relativistic effects destabilizing and expanding $d$ orbitals. The results for Mo and $\mathrm{W}$ were in good agreement with experimentally measured thermodynamic stabilities [11] and calculations from reference [9]. More recently, the physisorption interaction of group 6 hexacarbonyl species on quartz surfaces were quantified using relativistic density functional theory in conjunction with a physisorption model [12] (see Table 1). On-line gas-phase adsorption chromatography 
Table 1. Relevant calculated and experimentally determined thermodynamic data available for group 6 hexacarbonyl species: the standard adsorption enthalpy on quartz surfaces at zero surface coverage, $\Delta \mathrm{H}_{\text {ads }}(\mathrm{Quartz})$, and first bond dissociation enthalpy, FBDE.

\begin{tabular}{|l|c|c|c|c|}
\hline Element & $\begin{array}{c}\Delta \mathbf{H}_{\text {ads }}(\text { Quartz), } \\
\mathbf{k J} / \mathbf{m o l}[\mathbf{e x p} .] \\
{[\mathbf{1 5}]}\end{array}$ & $\begin{array}{c}\Delta \mathbf{H}_{\text {ads }}(\mathbf{Q u a r t z}), \\
\mathbf{k J} / \mathbf{m o l}[\text { theor.] } \\
{[\mathbf{1 2}]}\end{array}$ & $\begin{array}{c}\text { FBDE, } \\
\mathbf{k J} / \mathbf{m o l} \text { [exp] } \\
{[\mathbf{1 1}]}\end{array}$ & $\begin{array}{c}\text { FBDE, } \\
\mathbf{k J} / \mathbf{m o l}[\text { [theor.] } \\
{[\mathbf{1 0}]}\end{array}$ \\
\hline $\mathrm{Mo}$ & $49 \pm 2$ & $48.5 \pm 2$ & 169 & 169 \\
\hline $\mathrm{W}$ & $50 \pm 2$ & $46.5 \pm 2$ & 192 & 192 \\
\hline $\mathrm{Sg}$ & $50 \pm 2$ & $46.2 \pm 2$ & - & 208 \\
\hline
\end{tabular}

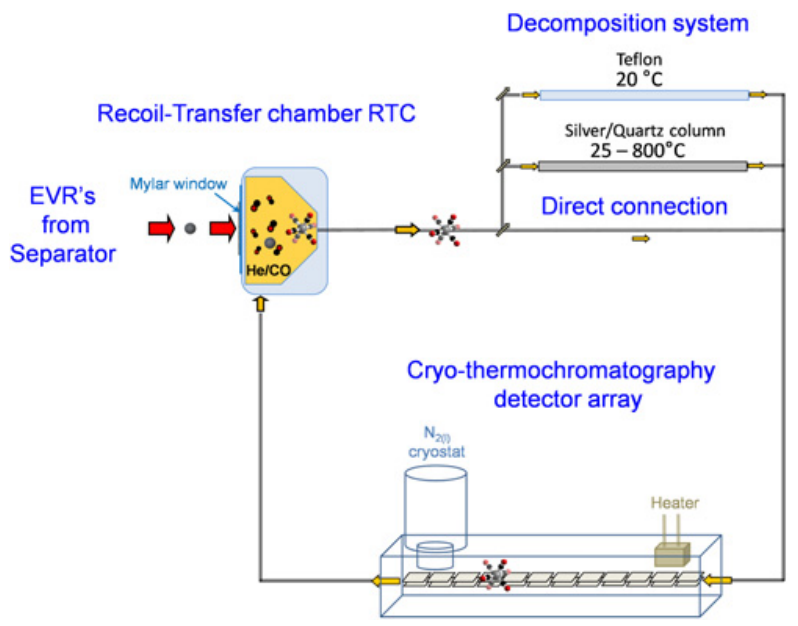

Figure 1. Scheme of the gas loop setup installed at the focal plane of a recoil separator for the online investigation of chemical properties of group 6 hexacarbonyl species regarding the formation, the adsorption, and thermal decomposition (for details see text).

chemistry experiments performed using radioactive isotopes of group 6 elements allowed for the determination of the adsorption interaction of these carbonyl species on quartz surfaces, pointing to a weak physisorption interaction, indeed, as expected by theory and from experiments with macroscopic amounts [4-8]. Thus, indirectly the speciation of the observed compounds was bolstered.

\subsection{The experimental observation of $\mathrm{Sg}(\mathrm{CO})_{6}$}

The typical scheme of experiments with single atoms behind gas-filled separators is shown in Fig. 1 for the case of the experiments using the GARIS separator at RIKEN Wako, Japan.

The general principle following this pre-separation scheme is described here: Recoiling nuclear fusion reaction products recoiling out of the target are separated according to their momentum-over-charge ratio and are guided by magnetic lens systems to the focal plane of the separator. There they pass through a thin Mylar window separating the (0.5 torr) He-gas filling of the separator from the gas-phase chemistry device starting with the Recoil Transfer Chamber (RTC). RTC is a closed chamber with gas inlets and an outlet mounted behind the Mylar window covering the entire area of this vacuum window. The RTC is flushed with gas mixtures of $\mathrm{He}$ and $\mathrm{CO}$ of variable content held at room temperature and at pressures typically between 0.1 and 1 bar. After passing this Mylar foil the products are thermalized within the volume of the RTC. Here, the in-situ formation reaction occurs. The formed volatile species are transported with the gas stream through thin PFA ${ }^{\circledR}$ Teflon capillaries with 


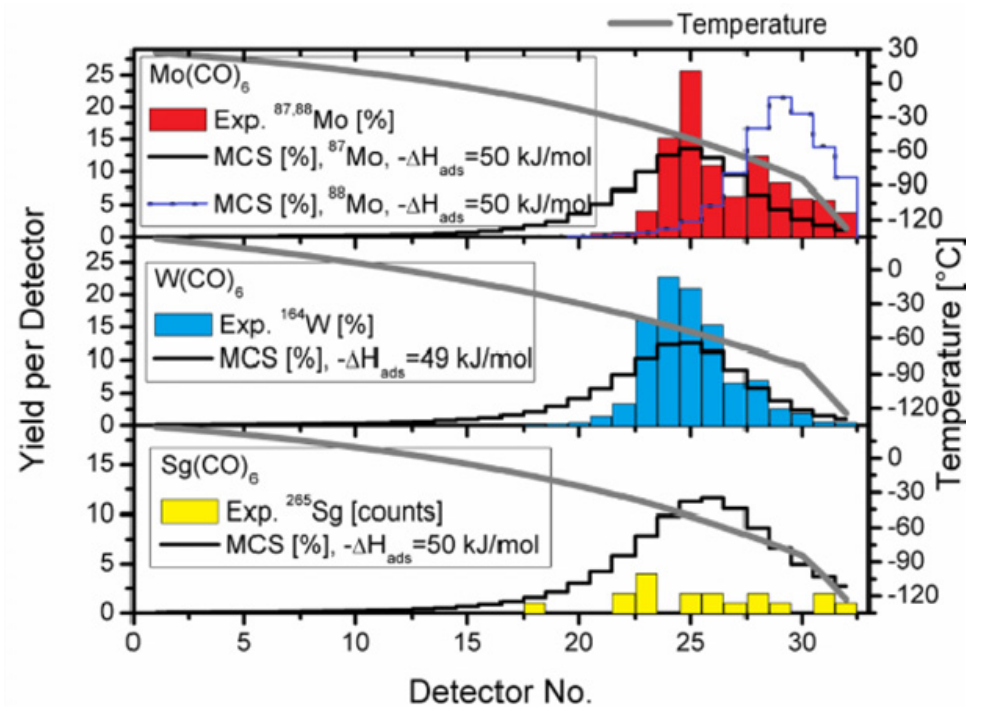

Figure 2. Thermochromatographic deposition patterns (bars) as measured for the group 6 hexacarbonyl compounds including $\mathrm{Sg}$ and revealing a very similar weak adsorption interaction of these species with quartz surfaces (adapted from Ref. [15]).

$2 \mathrm{~mm}$ inner diameter over the direct connection (see Fig. 1) to the detection system. Cryothermochromatography setups as used for the chemical investigations of group 8 oxides $\mathrm{MO}_{4}$ and for the investigation of volatile elements like $\mathrm{Hg}, \mathrm{Cn}$, and $\mathrm{Fl}$ (see reference [14] for a review) are best suited to study the in-situ chromatographic deposition of the formed volatile species. Using detector surfaces as chromatographic surfaces represents nowadays the most sensitive approach to detect single atomic adsorption. The detectors themselves are sensitive to the characteristic time correlated $\alpha$ and spontaneous fission decays. The following nuclear reactions were applied to produce single atomic isotopes of elements $\mathrm{Mo}, \mathrm{W}$, and $\mathrm{Sg}$ :

$$
\begin{aligned}
{ }^{\mathrm{nat}} \mathrm{Zn}\left({ }^{24} \mathrm{Mg}, \mathrm{x} n\right){ }^{87} \mathrm{Mo} \rightarrow & \left({ }^{87} \mathrm{Mo}: T_{1 / 2}=14 \mathrm{~s}, E_{\beta+}=5.3-5.5 \mathrm{MeV}\right) \\
{ }^{144} \mathrm{Sm}\left({ }^{24} \mathrm{Mg}, 4 n\right){ }^{164} \mathrm{~W} \rightarrow & \left({ }^{164} \mathrm{~W}: T_{1 / 2}=6.0 s, E_{\alpha}=5.15 \mathrm{MeV}\right) \\
{ }^{248} \mathrm{Cm}\left({ }^{22} \mathrm{Ne}, 5 n\right){ }^{265} \mathrm{Sg} \rightarrow & \left({ }^{265 \mathrm{a}} \mathrm{Sg}: T_{1 / 2}=8.5 s, E_{\alpha}=8.84 \mathrm{MeV} ;\right. \\
& \left.{ }^{265 \mathrm{~b}} \mathrm{Sg}: T_{1 / 2}=14.4 s, E_{\alpha}=8.69 \mathrm{MeV}\right) .
\end{aligned}
$$

The formation of volatile carbonyl species was observed and their deposition in the cryothermochromatography detector COMPACT $[14,15]$ was investigated. Example results are shown in Fig. 2. Based on the comparison with measurements performed using detector arrays, where all products passing the separator were implanted at the focal plane of the separator it was possible to estimate a formation and transport yield as $40 \%$ for ${ }^{87} \mathrm{Mo}$, and $10-20 \%$ for ${ }^{164} \mathrm{~W}$. Comparison of the number of events attributed to decays of ${ }^{265} \mathrm{Sg}$ observed in COMPACT with results from measurements studying this nuclear fusion reaction in detail [16] allowed for estimation of an overall chemical yield for $\mathrm{Sg}$ of about 5\% including the detection efficiency. The reasons for these low yields could so far not be understood. However, for the first time a transactinide complex compound featuring a metal-carbon bond as is characteristic of the organometallic compounds was investigated. 


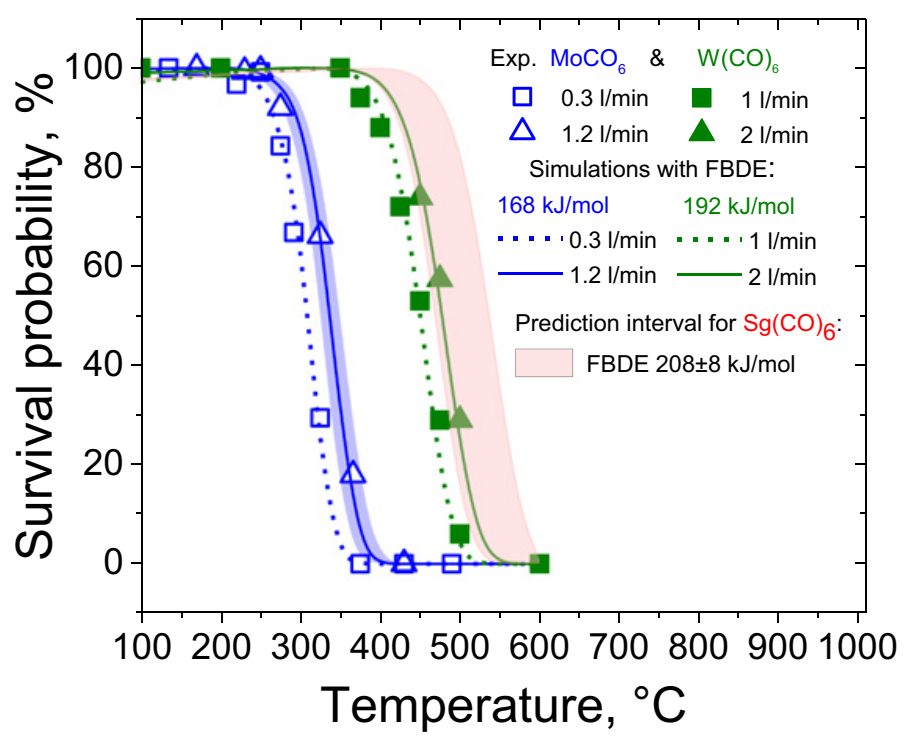

Figure 3. Experimental decomposition curves of $\mathrm{Mo}(\mathrm{CO})_{6}$ (blue symbols) and $\mathrm{W}(\mathrm{CO})_{6}$ (green symbols) at different gas flow rates. Simulated decomposition curves (lines) using for $\operatorname{Mo}(C O)_{6}:-\Delta \mathrm{H}_{\text {ads }}=48.8 \mathrm{~kJ} / \mathrm{mol}, \mathrm{FBDE}=169 \mathrm{~kJ} / \mathrm{mol}$; and for $\mathrm{W}(\mathrm{CO}) 6:-\Delta \mathrm{H}_{\mathrm{ads}}=47.5 \mathrm{~kJ} / \mathrm{mol}$, $\mathrm{FBDE}=192 \mathrm{~kJ} / \mathrm{mol}$. The blue shaded area shows the response of the model to $\Delta \mathrm{H}_{\mathrm{ads}}$ within uncertainties of $4 \mathrm{~kJ} / \mathrm{mol}$. The prediction interval within the uncertainty of $8 \mathrm{~kJ} / \mathrm{mol}$ for the FBDE of $\mathrm{Sg}(\mathrm{CO})_{6}$ given in Ref. [10] is shown as red shaded area.

\subsection{The stability of group 6 hexacarbonyl compounds $M(\mathrm{CO})_{6}$}

The observations described in the previous chapter paved the way towards the recently developed second-generation experiments with group 6 hexacarbonyls aiming for the detailed investigation of the stability of the metal carbon bond in those molecules. The relevant thermodynamic measure for this property is typically represented as the first carbonyl bond dissociation enthalpy (see Table 1, FBDE). Model experiments with isotopes of Mo emerging form a ${ }^{252} \mathrm{Cf}$ spontaneous fission source "Miss Piggy" installed at Bern University [17] were performed. It was shown in Ref. [18] that the simplest approach addressing the stability on a molecular level would be thermal decomposition studies on a silver surface. A typical decomposition setup used for this purpose comprised a tubular flow reactor of $1 \mathrm{~m}$ length and $4 \mathrm{~mm}$ inner diameter entirely covered inside with thin silver foils. The reactor could be heated to defined temperatures between room temperature and $600^{\circ} \mathrm{C}$. This reactor was placed between the carbonyl production side (for example, the RTC) and the detector for hexacarbonyl species (for example, COMPACT) (see the decomposition setup in Fig. 1). The amount of hexacarbonyl species able to pass this reactor in dependence of the selected isothermal temperature is measured in comparison to the total amount of hexacarbonyl species passing through a PFA ${ }^{\circledR}$ Teflon tube of the same geometry mounted as by-pass of the decomposition reactor as depicted in Fig. 1. The results obtained for $\mathrm{Mo}(\mathrm{CO})_{6}$ and $\mathrm{W}(\mathrm{CO})_{6}$ are shown in Fig. 3 (symbols). The observed shift of the decomposition curves of $\mathrm{W}(\mathrm{CO})_{6}$ of about $100^{\circ} \mathrm{C}$ relative to the curves measured for $\mathrm{Mo}(\mathrm{CO})_{6}$ confirm a higher stability of the tungsten complex as expected from Refs. [10,11]. A kinetic decomposition model was developed superimposing gas chromatographic transport of species through the open tubular reactor with heterogeneous decomposition of the species upon adsorption on the inner 
surfaces of the reactor [19]. This model was successfully applied to relate the decomposition observations to the FBDE given for the hexacarbonyl complexes [10, 11]. The obtained model describes the decomposition observations for Mo and $\mathrm{W}$ at varied experimental parameters very well (see Fig. 3, lines). Therefore, it was also applied in reference [19] to predict the hypothetical decomposition interval for $\operatorname{Sg}(\mathrm{CO})_{6}$ at the same experimental conditions (at $11 / \mathrm{min}$ gas flow rate) based on the theoretically predicted FBDE [10]. This prediction revealed the suggested method to be sensitive enough for a comparative study. Very recently, first decomposition experiments with ${ }^{265} \mathrm{Sg}(\mathrm{CO})_{6}$ have been performed successfully. The final data analysis is ongoing and will be presented elsewhere.

\section{Conclusions}

The availability of products of heavy ion induced nuclear fusion reactions pre-separated from the intense heavy ion beams paved the way towards the investigation of highly interesting fragile gas-phase complex compounds. The observation of volatile carbonyl complex formation for group 7-9 elements asks for the development of speciation techniques, for example based on mass spectrometry, to identify those volatile species.

Other organic ligands are known to form volatile gas-phase complexes with transition metals. Here the cyclopentadien forming ferrocene and ruthenocene shall be mentioned. The compound class of acetylacetonates with which this type of experiments have been started are still to be further explored. An interesting feature of the lighter 16 elements is the formation of gaseous "monocarbonyls" $\mathrm{MCO} M=\mathrm{S}$ [20], Se [21, 22], Te [23], though the bonding situation is different from that in the the complex compounds presented in this paper. However, in this chemical system the formation of volatile PoCO remains to be studied in preparation of future experiments with element 116 , livermorium.

\section{References}

[1] A. Türler and V. Pershina, Chem. Rev. 113, 1237 (2013).

[2] The Chemistry of Superheavy Elements, 2nd edn. (eds. M. Schädel and D. Shaughnessy, Springer, Berlin, 2014).

[3] Ch.E. Düllmann et al., Radiochim. Acta 97, 403 (2009).

[4] J. Even et al., Inorg. Chem. 51, 6431 (2012).

[5] J. Even et al., Radiochim. Acta 102, 1093 (2014).

[6] J. Even et al., J. Radioanal. Nucl. Chem. 303, 2457 (2015).

[7] Y. Wang et al., Radiochim. Acta 102, 69 (2014).

[8] Y. Wang, Z. Qin, F.-L. Fan, H. Haba, Y. Komori, S.-W Cao, X.-L. Wua, C.-M. Tana, Phys. Chem. Chem. Phys. 17, 13228 (2015).

[9] G. Frenking and N. Fröhlich, Chem. Rev. 100, 717 (2000).

[10] C.S. Nash and B.E. Bursten, J. Am. Chem. Soc. 121, 10830 (1999).

[11] K.E. Lewis, D.M. Golden, G.P. Smith, J. Am. Chem. Soc. 106, 3905 (1984).

[12] V. Pershina and J. Anton, J. Chem. Phys. 138, 174301 (2013).

[13] H. Haba et al., J. Nucl. Radiochem. Sci. 8(2), 55 (2007).

[14] A. Türler, R. Eichler, A. Yakushev, Nucl. Phys. A944, 640 (2015).

[15] J. Even et al., Science 345, 1491 (2014).

[16] H. Haba et al., Phys. Rev. C 85, 024611 (2012).

[17] Ch.E. Düllmann et al., Nucl. Instr. and Meth. A512, 595 (2003).

[18] I. Usoltsev et al., Radiochim. Acta 104, 141 (2016). 
[19] I. Usoltsev, R. Eichler, A. Türler, Radiochim. Acta, in press, DOI: 10.1515/ract-20152447 (2016).

[20] E.K.C. Lee, Y.N. Tang, F.S., Rowland, J. Phys. Chem., 68(2), 318 (1964).

[21] R.S. Baldwin, B.O. Peoto, L.B. Church, Radiochim. Acta 16(2), 94-96 (1971).

[22] M. Wachsmuth, B. Eichler, L. Tobler, D.T. Jost, H.W. Gäggeler, M. Ammann, Radiochim. Acta 88, 873 (2000).

[23] R.G. Strickert, S. Amiel, A.C. Wahl, Inorg. Nucl. Chem. Lett. 10, 129 (1974). 